\title{
Gestational Exposure to Pesticides Induces Oxidative Stress and Lipid Peroxidation in Offspring that Persist at Adult Age in an Animal Model
}

\author{
Elvis Ngwa Ndonwi ${ }^{1,2,3}$, Barbara Atogho-Tiedeu ${ }^{1,3}$, Eric Lontchi-Yimagou ${ }^{4}$, Tijjani S. Shinkafi ${ }^{2,5}$, \\ Dieudonne Nanfa ${ }^{1,3}$, Eric V. Balti ${ }^{6}$, Routray Indusmita ${ }^{2}$, Amena Mahmood ${ }^{2}$, Jean-Claude Katte ${ }^{7}$, \\ Armand Mbanya ${ }^{4}$, Tandi Matsha ${ }^{8}$, Jean Claude Mbanya ${ }^{1,7,9}$, Ali Shakir ${ }^{5}$ and Eugene Sobngwi ${ }^{1,7,9}$ \\ 'Laboratory for Molecular Medicine and Metabolism, Biotechnology Center, University of Yaoundé 1, Yaoundé, \\ Cameroon \\ ${ }^{2}$ Department of Biochemistry, Jamia Hamdard Deemed University, New-Delhi, India \\ ${ }^{3}$ Faculty of Science, University of Yaounde 1, Yaounde, Cameroon \\ ${ }^{4}$ Diabetes Research Center, Albert Einstein College of Medicine, New York, USA \\ ${ }^{5}$ Department of Biochemistry, Usmanu Danfodiyo University Sokoto, Sokoto, Nigeria \\ ${ }^{6}$ Diabetes Research Center, Brussels Free University-VUB, Brussels, Belgium \\ ${ }^{7}$ National Obesity Centre, Yaoundé Central Hospital, Yaoundé, Cameroon \\ ${ }^{8}$ Department of Biomedical Sciences, Faculty of Health and Wellness Sciences, Cape Peninsula University of \\ Technology, Cape Town, South Africa \\ ${ }^{9}$ Department of Internal Medicine, Faculty of Medicine and Biomedical Sciences, University of Yaoundé 1, Yaoundé, \\ Cameroon
}

\begin{abstract}
Pesticide exposure may induce biochemical alterations including oxidative stress and lipid peroxidation. However, in the context of developmental origin of health and disease, putative trans-generational effect of exposure to pesticides are insufficiently studied. We therefore aimed to evaluate the biochemical effect of gestational exposure to four pesticides on female Wistar rats and their offspring at adult age. We studied 30 female nulliparous Wistar rats divided into 5 equal groups. Group 1 served as the control group and received distilled water while group 2, 3, 4 and 5 received orally pesticide 1 (imidacloprid), pesticide 2 (chlorpyrifos), pesticide 3 (imidacloprid + lambda cyhalothrin) and pesticide 4 (oxamyl) respectively once daily throughout gestation at a dose equivalent to $1 / 10$ lethal dose 50 . The mothers were followed up until one month post gestation. The offspring were followed up from birth until adult age (12 weeks). In all animals at each time point we evaluated malondialdehyde (MDA), oxidative stress and liver function enzymes. There was similar variation of total body weight in all the groups during and after gestation. However, Female Wistar rats of the exposed groups had significant alterations in liver SOD $(-30.8 \%$ to $+64.1 \%)$, catalase $(-38.8 \%$ to $-85.7 \%)$ and GSH $(-29.2 \%$ to $-86.5 \%)$ and; kidney catalase (>100\%), GSH (>100\%). Moreover, MDA, alanine transaminase (ALT) and aspartate transaminase (AST) levels were significantly higher in pesticide exposed rats compared to the control group. Similar alterations in antioxidant enzymes, MDA and liver function enzymes were observed in offspring of treated rats evidenced at weaning and persisting until adult age. Exposure to
\end{abstract}

Correspondence to: Elvis Ngwa Ndonwi, Laboratory of Molecular Medicine and Metabolism, Faculty of Science University of Yaoundé 1, Yaounde 3851, Cameroon

E-mail: ngwaelvis73@yahoo.fr

This is an Open-Access article distributed under the terms of the Creative Commons Attribution Non-Commercial License (http:// creativecommons.org/licenses/by-nc/3.0) which permits unrestricted non-commercial use, distribution, and reproduction in any medium, provided the original work is properly cited.
Abbreviations: ALT, Alanine Transaminase; AST, Aspartate Transaminase; BSA, Bovine Serum Albumin; EC, Emulsifiable concentrate; EDTA, Ethylenediaminetetraacetic acid; GPx, Glutathione peroxidase; GR, Glutathione reductase; MDA, Malondialdehyde; NADP, Nicotinamide adenine dinucleotide phosphate; nAChR, Nicotinic acetylcholine receptors; OD, Optical Density; GSSG, Oxidized Glutathione; GSH, Reduced glutathione; SL, Soluble liquid; SEM, Standard error of mean; SOD, Superoxide dismutase. 
pesticides causes oxidative stress and lipid peroxidation in exposed female Wistar rats and their offspring. The persistence in offspring at adult age suggests transgenerational adverse effects.

Key words: Foetal exposure, Insecticide, Biochemical alterations, Offspring, Adult age

\section{INTRODUCTION}

Environmental contaminants especially pesticides are now responsible for the development of diseases both in wildlife and humans $(1,2)$. The recent classes of pesticides are organophosphate, carbamates, pyrethroids and neo-nicotinoids. Common pesticides belonging to these classes which are currently used are oxamyl, lambda cyhalothrin, chlorpyrifos and imidacloprid (3). The principal mechanisms of action of these pesticides include phosphorylation and inhibition of the action of acetylcholinesterase by organophosphates and carbamates, blocking of the open gates sodium channels by pyrethroids while neonicotinoids act by binding to the nicotinic acetylcholine receptors (nAChRs) and acting as nAChR agonists (4,5). In addition to their principal mode of action, lambda cyhalothrin, chlorpyrifos and imidacloprid have been reported to cause oxidative stress and lipid peroxidation both in vitro and in vivo (6-12). The degree of oxidative stress was enhanced with the combination of two pesticides $(13,14)$.

The effect of gestational exposure to maternal pesticides are understudied meanwhile there is increasing evidence on the role of various exposures during early life (pre-natal and post-natal) on adult metabolism and biochemical status. Moreover, investigators like Tukhtaev and collaborators reported that long-term exposure to lambda-cyhalothrin led to the development of oxidative stress in pregnant female rats and their offspring during breastfeeding (15). Whether the effect of this exposure could persist at adult age is unknown. We therefore undertook this study aiming at investigating whether biochemical alteration resulting from exposure to pesticides during pregnancy would persist at adult age. We studied alone and in combination, the most commonly used agricultural pesticides in Africa.

\section{MATERIALS AND METHODS}

Chemicals and reagents. L-Drint 20 [(20\% emulsifiable concentrate (EC) chlorpyrifos)], Confidor [(17.8\% soluble liquid (SL) imidacloprid)], Parastar (2\% imidacloprid + $2 \%$ lambda cyhalothrin) and Furaplant (10\% oxamyl) were purchased from Bayer Company (New Delhi, India). Kits for ALT and AST were purchased from ThermoFisher Scientific (New Delhi, India), while antioxidant enzymes and MDA quantification was carried out with biochemical reagents of molecular grade.

Animals and handling. Nulliparous Wistar rats, aged 10-12 weeks and weighing on average $210 \mathrm{~g}$, were used in this study. The animals were housed in the Animal House of Jamia Hamdard University in polypropylene cages under light controlled conditions with a 12/12-hr lightdark cycle and a temperature of $22 \pm 2^{\circ} \mathrm{C}$ and had free access to water and food. The study was approved by the Animal Ethics Committee of Jamia Hamdard University and the animals used in this study were treated humanely with regards for the alleviation of suffering.

\section{Experimental design.}

- Female rat sub-study: This was a prospective study with a follow-up period of 16 weeks. After acclimatization for a period of 7 days, the rats were fasted overnight and body weight was measured. Animals were then divided into 5 groups of six rats each (Group 1 to Group 5) matched with weight. In each group, animals were mated at a ratio of two females to each male in a cage. At gestation (vaginal swap smear and staining with methyl blue stain and examine for presence of sperms in the smear), the male rats were removed and, pesticide administration was initiated in all female rats. Female rats of Group 2, Group 3, Group 4 and Group 5 received pesticide 1 (44 mg imidacloprid per $\mathrm{kg}$ body weight/day), pesticide 2 ( $13.5 \mathrm{mg}$ chlorpyrifos per $\mathrm{kg}$ body weight/day), pesticide 3 (5.6 mg imidacloprid $+5.6 \mathrm{mg}$ lambda cyhalothrin per $\mathrm{kg}$ body weight/day) and pesticide $4(0.4 \mathrm{mg}$ oxamyl per kg body weight/day) respectively with gastroesophageal probe throughout gestation for 22 days while Group 1 received distilled water. These doses were chosen as shown to induce biochemical alterations without causing mortality and considered to be smaller than the exposed doses in humans during pesticide application, accidental poisoning (water or food containing pesticides). Each solution was freshly prepared by diluting in water and administered between 9 and $10 \mathrm{AM}$ at a dose volume of $10 \mathrm{~mL} / \mathrm{kg}$ rat per day. Pesticide administration was discontinued at birth while animals were maintained on normal diet and rats were followed for a month. At the end of follow up blood was collected by retro orbital puncture into ethylenediaminetetraacetic acid (EDTA) tubes, plasma was prepared and stored at $-20^{\circ} \mathrm{C}$ for ALT and AST quantification. After collection of blood samples, the rats were sacrificed by decapitation after local anesthesia and dissected. Liver and kidney were removed, washed in ice cold normal saline solution and homogenates prepared and refrigerated at $-80^{\circ} \mathrm{C}$ for quantification of antioxidant enzymes (SOD, catalase, GPx, GSH and GR) and MDA.

- Offspring sub-study: At birth, offspring were followed up by weekly recording of body weight. The off- 
spring were weaned at 4 weeks of age and were followed up until adult age (12 weeks). At adult age, 8 offspring (4 male and 4 female) from each group were randomly selected and sacrificed at the end of follow up. Blood was collected and plasma prepared for ALT and AST quantification while liver and kidney samples were collected for antioxidant enzymes and MDA quantification.

\section{Biochemical assays.}

- Super-oxide dismutase quantification: Superoxide dismutase (SOD) was assayed using the method of Marklund and Marklund (16) with slight modifications. A single unit of enzyme was expressed as $50 \%$ inhibition of pyrogallol reduction $/ \mathrm{min} / \mathrm{mg}$ protein by superoxide is measured at $420 \mathrm{~nm}$. Briefly, $0.1 \mathrm{~mL}$ of the sample solution (liver or kidney homogenate) was added to $2.8 \mathrm{~mL}$ of Tris-EDTA buffer solution in a cuvette, $0.1 \mathrm{~mL}$ of pyrogallol was added. The solution was mixed rapidly by inversion and the optical density was measured at $420 \mathrm{~nm}$ using the kinetic programme (30 sec interval for $3 \mathrm{~min}$ ). A control tube was also prepared replacing the sample with $0.1 \mathrm{~mL}$ doubled distilled water. Enzyme activity was expressed as units/mg protein.

- Catalase quantification: Catalase was assayed using the method of Aebi (17). Briefly, samples were diluted in the ratio 1:50 with phosphate buffer. One $\mathrm{mL}$ of hydrogen peroxide $(60 \mathrm{mM})$ was added into the diluted samples, mixed rapidly by inversion and the optical density read at $420 \mathrm{~nm}$ at $30 \mathrm{sec}$ interval for $2 \mathrm{~min}$.

Enzymatic activity was expressed as $\mu$ moles of $\mathrm{H}_{2} \mathrm{O}_{2}$ decomposed $/ \mathrm{min} / \mathrm{mg}$ protein using molar extinction coefficient of $\mathrm{H}_{2} \mathrm{O}_{2}\left(43.6 \mathrm{M}^{-1} \mathrm{~cm}^{-1}\right)$.

- Glutathione peroxidase quantification: This assay was carried out using the method of Wendel (18). Briefly, the reaction mixture $(7.0 \mathrm{mg}$ of sodium azide, $33.2 \mathrm{mg}$ reduced glutathione (GSH), and $1.08 \mathrm{mg}$ reduced NADPH in $100 \mathrm{~mL}$ of phosphate buffer) was prepared and $3 \mathrm{~mL}$ added into the cuvette followed by $50 \mu \mathrm{L}$ of hydrogen peroxide $(0.042 \%)$. Fifty $\mu \mathrm{L}$ of sample was then added, rapidly mixed by inversion and the optical density read at $340 \mathrm{~nm}$ at $30 \mathrm{sec}$ for $3 \mathrm{~min}$. The results were expressed as nmoles NADPH oxidized $/ \mathrm{min} / \mathrm{mg}$ protein, using molar extinction coefficient of NADPH $\left(6.22 \times 10^{6} \mathrm{M}^{-1} \mathrm{~cm}^{-1}\right)$.

- Reduced glutathione quantification: This assay was carried out using the method of Moron (19). Briefly $200 \mu \mathrm{L}$ of samples were diluted to $1,000 \mu \mathrm{L}$ with phosphate buffer and reduced Glutathione standards were prepared. A blank tube was equally prepared containing $1 \mathrm{~mL}$ of double distilled water. Into each of the tubes (blank standard and samples) was added $1 \mathrm{~mL}$ of Ellman's Reagent solution. Each of the tube was incubated and the optical density (OD) read between 2 and $10 \mathrm{~min}$ at $412 \mathrm{~nm}$ in a spectrophotometer. A graph of $\mathrm{OD}=\mathrm{f}$ (concentration) of standard was used to determine the concentration of GSH in samples by extrapolation and expressed in nmole.
- Glutathione reductase quantification: This assay was carried out using the method of Mavis and Stellwagen (20). Briefly, $2.9 \mathrm{~mL}$ of the reaction mixture $(0.65 \mathrm{~mL}$ distilled water, $1.5 \mathrm{~mL}$ phosphate buffer $100 \mathrm{mM}$ pH 7.0, $0.1 \mathrm{~mL}$ GSSG $30 \mathrm{mM}, 0.35 \mathrm{~mL} \beta$-NADPH $0.8 \mathrm{mM}$ and $0.3 \mathrm{~mL} 1 \% \mathrm{w} / \mathrm{v}$ BSA) was introduced into the cuvette followed by $0.1 \mathrm{~mL}$ of sample and mixed rapidly by inversion. The optical density was then read at $340 \mathrm{~nm}$ using a kinetic program (30 sec for $3 \mathrm{~min}$ ). The activity of the enzyme was expressed as nmoles NADPH oxidized/min/ $\mathrm{mg}$ protein, using molar extinction coefficient of NADPH $\left(6.22 \times 10^{6} \mathrm{M}^{-1} \mathrm{~cm}^{-1}\right)$.

- Malondialdehyde quantification: Malondialdehyde (MDA) was quantified as thiobarbituric acid reactive substrate using the method modified from that of Ohkawa (21). One hundred microliters of each sample was added into a Pyrex test tube followed by $400 \mu \mathrm{L}$ of the thiobarbituric acid substrate reagent mixture and the tubes were closed tightly. The mixture was heated at $100^{\circ} \mathrm{C}$ for $15 \mathrm{~min}$. After heating the cork was removed and the tubes were placed in an ice bath for cooling to occur. After the cooling process, the mixture was centrifuged at $1,500 \mathrm{~g}$ for $5 \mathrm{~min}$. The optical density of the supernatant was read at $532 \mathrm{~nm}$. The concentration of MDA was then calculated using the molar extinction coefficient of MDA $(\varepsilon=$ $1.56 \times 10^{5} \mathrm{M}^{-1} \mathrm{~cm}^{-1}$ ) as follows:

$$
\begin{aligned}
& \text { Concentration of } \operatorname{MDA}(\mu \mathrm{M}) \\
& \qquad=\frac{\mathrm{OD}}{\varepsilon} \times 1,000,000 \times \text { dilution factor }
\end{aligned}
$$

- Total protein quantification: Total protein content in tissue samples was determined by the method of Lowry (22), using bovine serum albumin as the standard.

- Alanine transaminase and aspartate transaminase quantification: Alanine transaminase (ALT) and aspartate transaminase (AST) were quantified with ALT and AST reagent kits according to the manufacturer's protocol (ThermoFisher Scientific) in the plasma.

- Statistical analysis: The results were analyzed using GraphPad Software (GraphPad Software, CA, USA). Unpaired $t$-test followed by Welch's correction was used to compare data between control and exposed groups. The data obtained were expressed as mean \pm standard error of mean (SEM). All analyses were carried out at $95 \%$ confidence interval and $p<0.05$ was considered to be statistically significant.

\section{RESULTS}

Reproductive outcome, weight variation and liver function enzymes level in parent rats. The weights of the dams were similar in all groups throughout the study (Fig. 1). The average gestation length was not signifi- 


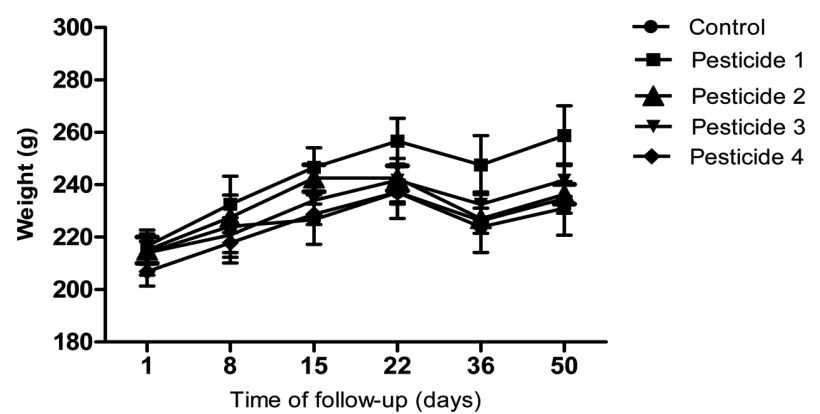

Fig. 1. Effect of pesticides exposure on body weight on female Wistar rats. Pesticide $1=$ imidacloprid, pesticide $2=$ chlorpyrifos, pesticide $3=$ imidacloprid + lambda cyhalothrine, pesticide $4=$ oxamyl. Data are reported as mean \pm standard error of mean (SEM) for 6 samples.

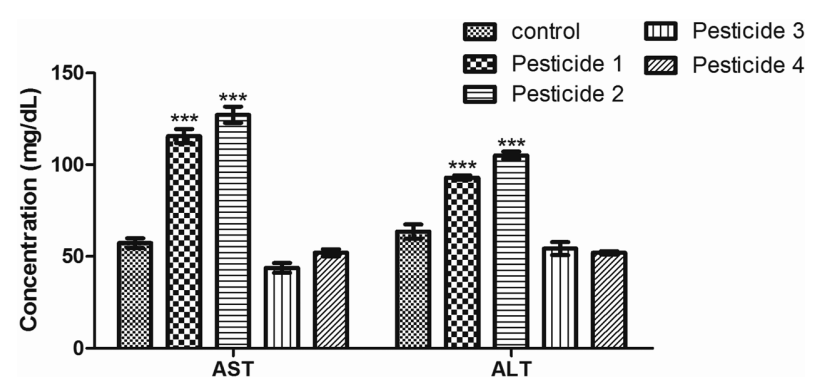

Fig. 2. Effect of pesticides on aspartate transaminase and alanine transaminase levels in female Wistar rats. Pesticide 1 = imidacloprid, pesticide $2=$ chlorpyrifos, pesticide $3=$ imidacloprid + lambda cyhalothrine, pesticide $4=$ oxamyl, ${ }^{* * *} p<$ 0.001 compared with normal control. Data are reported as mean \pm standard error of mean (SEM) for 8 samples.

cantly different between the control group ( $22 \pm 1$ day) and imidacloprid (23 \pm 2 days), chlorpyrifos ( $22 \pm 1$ day), lambda cyhalothrin + imidacloprid ( $22 \pm 2$ days) and oxamyl ( $24 \pm 3$ days) exposed groups. However, parent rats exposed to imidacloprid and chlorpyrifos had a significant increase in aspartate transaminase $(>100 \%)$ and alanine transaminase ( $46.4 \%$ for pesticide 1 and $65.5 \%$ for pesticide 2 ) lev- els compared to control (Fig. 2).

Antioxidant enzyme and malondialdehyde variation in liver and kidney of parent rats. Alterations in the level of antioxidant enzymes were recorded in the liver and kidney of parent rats exposed to pesticides when compared with the control group (Table 1). Liver SOD was significantly reduced in rats exposed to pesticide $1(30.8 \%)$, pesticide $2(38.5 \%)$ and $3(51.3 \%)$ while a significantly higher value was recorded in rats exposed to pesticide 4 (64.1\%) when compared with the control group. Exposure to all four pesticides led to a significant decrease in liver catalase and liver GSH of parent rats. Pesticide 1 and 2 significantly increase liver glutathione peroxidase (GPx) levels (50\% and $64.9 \%$ respectively) while pesticide 3 and 4 significantly reduced liver GPx levels (81.1\% and 83.9\% respectively). Liver glutathione reductase (GR) was significantly reduced in rats exposed to pesticide $4(64.5 \%)$ while liver MDA was significantly increased in rats exposed to pesticide 2 (>100\%). In the kidney, SOD was significantly increased in rats exposed to pesticide 1 $(100 \%)$ while GPx was significantly increased in rats exposed to pesticide $4(31.7 \%)$. Similarly, GSH was significantly increased in rats exposed to pesticide $1(52.8 \%)$ and pesticide $2(45.6 \%)$. Moreover, rats exposed to pesticide 1, 3 and 4 had a significantly higher MDA ( $>100 \%$ in all cases) levels when compared to control. Kidney catalase levels were significantly higher in rats exposed to pesticide 3 and 4 (> 100\% in both cases) while kidney GR levels were significantly lower in rats exposed to pesticide $3(38.4 \%)$ and $4(44.8 \%)$ compared to the control group.

Weight variation and liver function enzymes level in offspring. In offspring of pesticide exposed rats there was no significant different in body weight throughout the follow when compared with the control group (Fig. 3). However, offspring of rats exposed to all pesticides had a significant increase in ALT and AST levels when compared with the control group (Fig. 4).

Table 1. Effect of pesticides on antioxidant enzymes and malondialdehyde levels in liver and kidney of female Wistar rats

\begin{tabular}{|c|c|c|c|c|c|c|c|c|c|c|}
\hline \multirow{2}{*}{ Sample } & \multicolumn{5}{|c|}{ Liver } & \multicolumn{5}{|c|}{ Kidney } \\
\hline & Control & Pesticide 1 & Pesticide 2 & Pesticide 3 & Pesticide 4 & Control & Pesticide 1 & Pesticide 2 & Pesticide 3 & Pesticide 4 \\
\hline SOD & $3.9 \pm 0.4$ & $2.7 \pm 0.3^{*}$ & $2.4 \pm 0.2^{*}$ & $1.9 \pm 0.4^{* *}$ & $6.4 \pm 0.4^{* *}$ & $1.4 \pm 0.3$ & $2.8 \pm 0.4^{*}$ & $2.0 \pm 0.3$ & $1.3 \pm 0.2$ & $1.2 \pm 0.2$ \\
\hline CAT & $24.5 \pm 1.9$ & $14.7 \pm 1.4^{* *}$ & $15.0 \pm 1.7^{* *}$ & $3.5 \pm 0.8^{* * *}$ & $12.7 \pm 1.9^{* *}$ & $14.9 \pm 6.4$ & $22.4 \pm 0.9$ & $31.7 \pm 4.6$ & $35.9 \pm 1.9^{*}$ & $32.9 \pm 2.5^{*}$ \\
\hline GPx & $14.8 \pm 1.2$ & $22.2 \pm 1.9^{*}$ & $24.4 \pm 2.8^{*}$ & $2.8 \pm 0.5^{* * *}$ & $2.4 \pm 0.1 * * *$ & $20.8 \pm 2.2$ & $20.6 \pm 1.4$ & $21.1 \pm 1.1$ & $15.54 \pm 2.6$ & $14.2 \pm 1.4^{*}$ \\
\hline GSH & $26.7 \pm 2.4$ & $18.9 \pm 1.9^{*}$ & $17.3 \pm 2.4^{*}$ & $3.8 \pm 0.2 * * *$ & $3.6 \pm 0.3^{* * *}$ & $19.3 \pm 1.4$ & $29.5 \pm 1.7 * *$ & $28.1 \pm 2.9^{*}$ & $15.0 \pm 1.8$ & $16.8 \pm 1.6$ \\
\hline GR & $3.1 \pm 0.5$ & $1.7 \pm 0.6$ & $1.9 \pm 0.5$ & $2.2 \pm 0.4$ & $1.1 \pm 0.3^{*}$ & $48.4 \pm 3.4$ & $50.1 \pm 2.7$ & $48.5 \pm 3.0$ & $29.8 \pm 4.9^{*}$ & $26.7 \pm 3.1^{* *}$ \\
\hline MDA & $0.7 \pm 0.06$ & $5.3 \pm 0.3$ & $5.9 \pm 0.6^{* * *}$ & $0.6 \pm 0.1$ & $0.6 \pm 0.1$ & $1.0 \pm 0.1$ & $2.4 \pm 0.3^{*}$ & $1.6 \pm 0.3$ & $3.5 \pm 0.6^{* * *}$ & $5.0 \pm 0.3^{* * *}$ \\
\hline
\end{tabular}

Pesticide 1 = imidacloprid, pesticide $2=$ chlorpyrifos, pesticide $3=$ imidacloprid + lambda cyhalothrine, pesticide $4=$ oxamyl, SOD = super oxide dismutase, $\mathrm{CAT}=$ Catalase, $\mathrm{GPx}=$ Glutathione peroxidase, $\mathrm{GSH}=$ Reduced Glutathione, $\mathrm{GR}=$ Glutathione Reductase, ${ }^{*} p<0.05$, ${ }^{* *} p<0.01,{ }^{* * *} p<0.001$ compared with normal control. Data are reported as mean \pm standard error of mean (SEM) for 6 samples. 


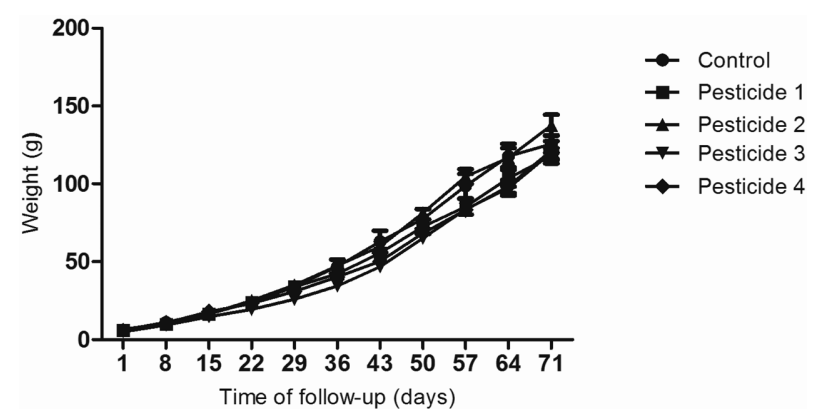

Fig. 3. Effect of pesticides on body weight in offspring. Pesticide $1=$ imidacloprid, pesticide $2=$ chlorpyrifos, pesticide $3=$ imidacloprid + lambda cyhalothrine, pesticide $4=$ oxamyl. Data are reported as mean \pm standard error of mean (SEM) for 8 samples.

\section{Antioxidant enzyme and malondialdehyde variation} in liver and kidney of offspring. Similar alterations in liver and kidney antioxidants and MDA levels were recorded in offspring of pesticide exposed rats when compared to the control group at weaning (Table 2) and persisted until adulthood (Table 3). Liver SOD was significantly lower in offspring of rats exposed to pesticide $1(87.6 \%)$ and pesticide $2(84.9 \%)$. Liver catalase was significantly higher in offspring of rats exposed to pesticide $1(30.3 \%)$ and pesti-

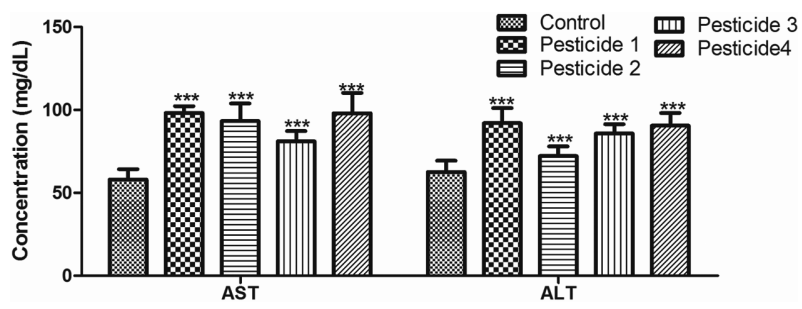

Fig. 4. Effect of pesticides on aspartate transaminase and alanine transaminase levels in offspring of female Wistar rats. Pesticide 1 = imidacloprid, pesticide $2=$ chlorpyrifos, pesticide $3=$ imidacloprid + lambda cyhalothrine, pesticide $4=$ oxamyl, ${ }^{* *}=$ $p<0.0001$ compared with normal control. Data are reported as mean \pm standard error of mean for 8 samples.

cide $2(36.9 \%)$ while a significantly lower catalase level (39.4\%) was recorded in offspring of rats exposed to pesticide 4. GPx level was significantly reduced in offspring of rats exposed pesticide $1(77.9 \%)$, pesticide $2(78.1 \%)$ and pesticide $4(30.3 \%)$ when compared to the control group. Similarly, liver GSH was significantly lower in offspring of rats exposed to pesticide 1 (30.4\%), pesticide $3(91.2 \%)$ and 4 (90.9\%). Likewise, a significantly lower liver GR was recorded in offspring of parent rats exposed to pesticide $3(41.4 \%)$ and $4(34.5 \%)$. A one-fold increase in liver

Table 2. Effect of pesticides on antioxidant enzymes and malondialdehyde levels in liver and kidney of offspring Wistar rats sacrificed at aweaning

\begin{tabular}{|c|c|c|c|c|c|c|c|c|c|c|}
\hline \multirow{2}{*}{ Sample } & \multicolumn{5}{|c|}{ Liver } & \multicolumn{5}{|c|}{ Kidney } \\
\hline & Control & Pesticide 1 & Pesticide 2 & Pesticide 3 & Pesticide 4 & Control & Pesticide 1 & Pesticide 2 & Pesticide 3 & Pesticide 4 \\
\hline SOD & $1.9 \pm 0.2$ & $1.7 \pm 0.2$ & $1.9 \pm 0.2$ & $1.3 \pm 0.2^{*}$ & $1.3 \pm 0.2^{*}$ & $2.8 \pm 0.6$ & $5.0 \pm 0.4^{*}$ & $5.7 \pm 0.6^{* *}$ & $4.6 \pm 1.1$ & $2.9 \pm 0.5$ \\
\hline CAT & $9.2 \pm 0.9$ & $14.1 \pm 1.8^{*}$ & $8.6 \pm 0.7$ & $11.9 \pm 2.5$ & $13.4 \pm 5.8$ & $10.7 \pm 5.6$ & $24.6 \pm 13.7$ & $48.9 \pm 29.4$ & $8.0 \pm 1.0$ & $8.3 \pm 0.8$ \\
\hline GPx & $28.2 \pm 2.3$ & $29.8 \pm 4.9$ & $26.7 \pm 3.1$ & $27.2 \pm 2.3$ & $33.5 \pm 1.4$ & $4.2 \pm 0.5$ & $13.4 \pm 5.8$ & $11.9 \pm 2.5^{*}$ & $7.0 \pm 2.2$ & $8.3 \pm 0.9 * *$ \\
\hline GSH & $21.4 \pm 3.3$ & $15.0 \pm 1.8$ & $16.8 \pm 1.6$ & $15.5 \pm 2.6$ & $14.2 \pm 1.4$ & $33.5 \pm 1.4$ & $69.1 \pm 6.5^{* *}$ & $75.7 \pm 4.9^{* * *}$ & $52.0 \pm 5.9^{*}$ & $53.2 \pm 7.0^{*}$ \\
\hline GR & $7.1 \pm 3.7$ & $1.8 \pm 0.3$ & $1.4 \pm 0.4$ & $1.3 \pm 0.2$ & $1.2 \pm 0.2$ & $3.3 \pm 0.4$ & $11.1 \pm 2.4^{*}$ & $6.9 \pm 1.4$ & $6.1 \pm 1.2$ & $6.8 \pm 1.2^{*}$ \\
\hline MDA & $3.5 \pm 0.4$ & $3.5 \pm 0.6$ & $5.0 \pm 0.3 *$ & $4.4 \pm 1.1$ & $3.3 \pm 0.4$ & $0.3 \pm 0.02$ & $0.9 \pm 0.2^{*}$ & $1.6 \pm 0.1^{* * *}$ & $0.9 \pm 0.1^{*}$ & $0.8 \pm 0.1^{* *}$ \\
\hline
\end{tabular}

Pesticide 1 = imidacloprid, pesticide $2=$ chlorpyrifos, pesticide $3=$ imidacloprid + lambda cyhalothrine, pesticide $4=$ oxamyl, SOD = super oxide dismutase, $\mathrm{CAT}=$ Catalase, $\mathrm{GPx}=$ Glutathione peroxidase, $\mathrm{GSH}=$ Reduced Glutathione, $\mathrm{GR}=$ Glutathione Reductase, ${ }^{*} p<0.05$, ${ }^{* *} p<0.01,{ }^{* * *} p<0.001$ compared with normal control. Data are reported as mean \pm standard error of mean (SEM) for 8 samples.

Table 3. OEffect of pesticides on antioxidant enzymes and malondialdehyde levels in liver and kidney of offspring Wistar rats at adulthood

\begin{tabular}{|c|c|c|c|c|c|c|c|c|c|c|}
\hline \multirow{2}{*}{ Sample } & \multicolumn{5}{|c|}{ Liver } & \multicolumn{5}{|c|}{ Kidney } \\
\hline & Control & Pesticide 1 & Pesticide 2 & Pesticide 3 & Pesticide 4 & Control & Pesticide 1 & Pesticide 2 & Pesticide 3 & Pesticide 4 \\
\hline SOD & $23.3 \pm 3.8$ & $2.9 \pm 0.3^{* *}$ & $3.5 \pm 0.4^{* *}$ & $19.3 \pm 1.4$ & $16.6 \pm 1.0$ & $3.8 \pm 0.3$ & $3.1 \pm 0.2$ & $3.4 \pm 0.3$ & $5.0 \pm 0.4^{*}$ & $5.7 \pm 0.6^{*}$ \\
\hline CAT & $16.5 \pm 1.0$ & $21.5 \pm 1.2^{* *}$ & $22.6 \pm 0.7 * * *$ & $17.9 \pm 2.5$ & $10.0 \pm 2.2^{*}$ & $17.4 \pm 2.5$ & $21.2 \pm 1.5$ & $26.9 \pm 2.9^{*}$ & $24.6 \pm 13.7$ & $48.9 \pm 29.4$ \\
\hline GPx & $70.9 \pm 4.4$ & $15.7 \pm 1.9^{* * *}$ & $15.5 \pm 1.3^{* * *}$ & $55.7 \pm 8.7$ & $49.4 \pm 4.5^{* *}$ & $7.5 \pm 0.6$ & $10.4 \pm 0.5^{* *}$ & $12.5 \pm 0.8^{* * *}$ & $13.4 \pm 5.8$ & $11.9 \pm 2.5$ \\
\hline GSH & $30.9 \pm 3.3$ & $21.5 \pm 1.9^{*}$ & $24.3 \pm 1.7$ & $2.7 \pm 0.2^{* * *}$ & $2.8 \pm 0.2^{* * *}$ & $26.4 \pm 3.3$ & $21.5 \pm 1.6$ & $14.6 \pm 3.3^{*}$ & $17.1 \pm 4.7$ & $20.7 \pm 4.1$ \\
\hline GR & $2.9 \pm 0.4$ & $2.5 \pm 0.6$ & $1.9 \pm 0.3$ & $1.7 \pm 0.3^{*}$ & $1.9 \pm 0.2^{*}$ & $31.8 \pm 1.2$ & $34.3 \pm 1.9$ & $37.1 \pm 1.4^{*}$ & $13.2 \pm 1.6^{* * *}$ & $12.6 \pm 1.4^{* * *}$ \\
\hline MDA & $5.8 \pm 1.4$ & $10.0 \pm 1.2^{*}$ & $10.3 \pm 0.9^{*}$ & $5.6 \pm 0.1$ & $6.1 \pm 0.2$ & $2.2 \pm 0.2$ & $3.2 \pm 0.3^{*}$ & $4.8 \pm 0.8^{*}$ & $2.4 \pm 0.5$ & $2.6 \pm 0.5$ \\
\hline
\end{tabular}

Pesticide 1 = imidacloprid, pesticide $2=$ chlorpyrifos, pesticide $3=$ imidacloprid + lambda cyhalothrine, pesticide $4=$ oxamyl, SOD $=$ super oxide dismutase, GPx = Glutathione peroxidase, GST = Glutathione $S$ transferase, GSH = Reduced Glutathione, GR= Glutathione Reductase, ${ }^{*} p<0.05,{ }^{* *} p<0.01,{ }^{* * *} p<0.001$ compared with normal control. Data are reported as mean \pm standard error of mean (SEM) for 8 samples. 
MDA was recorded in rats exposed to pesticide 1 (72.4\%) and $2(77.6 \%)$.

In kidney, SOD was significantly increased in offspring of rats exposed to pesticide $3(31.6 \%)$ and pesticide 4 $(50 \%)$. Kidney catalase was significantly higher $(54.6 \%)$ while kidney GSH was significantly lower (44.7\%) in offspring of parent rats exposed to pesticide 2. Moreover, kidney GPx and MDA levels were significantly higher in offspring of parent rats exposed to pesticide 1 (38.7\% and $45.5 \%$ for GPx and MDA respectively) and pesticide 2 (66.7\% and $118 \%$ for GPx and MDA respectively) when compared to the control group. GR was significantly higher in offspring of rats exposed to pesticide $2(16.7 \%)$ and significantly reduced in offspring of rats exposed to pesticide $3(58.5 \%)$ and pesticide $4(60.4 \%)$.

\section{DISCUSSION}

Pesticide poisoning constitute a public health problem as 3,000,000 cases of poisoning and 220,000 deaths were recorded annually across the globe, the majority of which are reported from developing countries (23). Pesticide usage/hectare (ha) is less in Africa (1.23 kg a.i./ha) compared to Latin America and Asia (23). However, the concern in Africa is alarming due to poor status of legislation, insufficient education and training in pesticide usage amongst others. In Cameroon, pesticide exposure mainly occur in agricultural sector, practiced by local farmers or industrialized companies such as Cameroon Development Corporation (CDC), a major development partner in Cameroon which cultivates rubber, oil palm and banana (24). Lack of respect of pesticide application measures, high run off of pesticides into nearby water sources as a result of aircraft spraying and dumping of pesticide waste amongst others result to pesticide poisoning in Cameroon (25). This has as consequence, killing of aquatic organisms, intoxication of the applicators and local population which can result to dead.

Organophosphate, carbamates and neonicotinoids insecticides as well as fungicides are extensively used in agricultural settings in Cameroon with inhalation and ingestion being the main exposure routes (25-27). This study is therefore relevant as it investigated pesticides which are widely used especially for agricultural purposes as well as in public health disease control. Rats were exposed to a dose assumed to be same or inferior to human exposed dose in agricultural setting with similar mode of exposure in humans. Investigation of parameters in offspring was essential so as to depict possible transgenerational effect. The present study shows that all the pesticides tested induced oxidative stress in female Wistar rats, characterised by alterations in circulating levels of at least one antioxidant enzyme in liver and kidney tissues. The observed increase in lipid peroxidation as well as high plasma ALT and AST levels in rats exposed to imidacloprid and chlorpyrifos were confirmatory of tissue damage. Similar alterations observed in parents were equally recorded in offspring at adult age. Oxidative stress in liver and kidney were observed in offspring of rats exposed to pesticides when compared to the control group characterized by alterations in antioxidant levels. Moreover imidacloprid and chlorpyrifos were shown to induce lipid peroxidation in offspring of rats exposed to these two chemicals. In a similar manner, in utero exposure to all 4 pesticides induced liver injury as observed by a 3 fold increase in plasma ALT and AST when compared to the control group.

Our findings in directly exposed animals are in accordance with most previous studies. Lambda-cyhalothrin was observed to induced oxidative stress both in vivo and in vitro (6-9). It significantly increased liver and kidney malondialdehyde (MDA) of male adult Wistar rats $(9,10)$. Similarly dysregulation in oxidative stress and lipid peroxidation biomarkers in rats exposed to imidacloprid was reported $(11,28-30)$. Furthermore, chlorpyrifos was observed to induce oxidative stress as well as increase levels of lipid peroxides in the (12,31-35). Notwithstanding, ALT and/or AST levels were significantly increased in plasma of exposed rats and their offspring $(36,37)$. However, the pesticide mixture $(5.6 \mathrm{~g} / \mathrm{kg}$ imidacloprid $+5.6 \mathrm{~g} / \mathrm{kg}$ lambda cyhalothrine) did not affect the liver function enzymes which can be explained by the low dose.

The mechanism of generation of oxidative stress is related to pesticide biotransformation as in the case of chlorpyrifos. Biotransformation of chlorpyrifos by cytochrome P450 generate its metabolites chlorpyrifos oxon and 3,5,6-trichloro-2-pyridinol. These metabolites being more toxic than the parent compound directly or indirectly generate reactive oxygen species such as highly reactive superoxide radical and hydrogen peroxide (38). At the onset, there is an increase in the level of antioxidants to counteract the effect of these ROS. However, prolonged exposure leads to depletion of the antioxidant system leading to cellular damage (39) and oxidative stress. The end stage of oxidative stress is impairment of cellular functions and potential tissue damage which is characterized by lipid peroxidation (increased MDA levels) and leakage of cytosolic enzymes from hepatocytes (ALT and AST).

What is new in our findings is the effect on offspring with in utero exposure that can be evidenced at weaning (Table 3) persist with similar magnitude at adult age without additional exposure. Tukhtaev et al. (15) observed similar alterations in oxidative stress enzymes in offspring of rats with inutero exposure to pesticides. However, they reported that the alterations decreased both in exposed rats and their offspring with cessation of breast milk. The difference could be because of the dose used since that in our study was 10 times higher. Therefore, pesticide exposure at 
certain doses could induce permanent alteration in exposed organisms. Even though pesticides were not quantified in body fluids of offspring, we can affirm that alterations in offspring resulted from foetal exposure since these chemicals have been shown to cross the placenta. Therefore, further studies looking at the epigenome to investigate the molecular mechanism as well as the second and third generation to characterize the trans-generational effect are warranted.

Commonly used pesticides in Africa (imidacloprid, chlorpyrifos, lambda cyhalothrin and oxamyl) induced oxidative stress, lipid peroxidation and caused liver injury in female Wistar rats and their offspring. These biochemical alterations on offspring remain until adult age suggesting that these chemicals persist in the offspring even after cessation of exposure or modify the epigenome through epigenetic mechanisms. Therefore, further studies looking at the epigenome to investigate the molecular mechanism are warranted.

\section{ACKNOWLEDGMENTS}

Appreciations go to Jamia Hamdard University (Department of Biochemistry and Animal Facility) for provision of space and equipments.

\section{CONFLICT OF INTEREST}

The authors declare that there are no conflicts of interest.

Received July 29, 2018; Revised September 12, 2018; Accepted October 30, 2018

\section{REFERENCES}

1. Ming, Y., Jeremy, B., Jonathan, W.M. and Ambikaipakan, S. (2013) Occupational pesticide exposures and respiratory health. Int. J. Environ. Res. Public Health, 10, 6442-6471.

2. Shin, H.J., Cho, H.G., Park, C.K., Park, K.H. and Lim, H.B. (2017) Comparative in vitro biological toxicity of four kinds of air pollution particles. Toxicol. Res., 33, 305-313.

3. Food and Agriculture Organization of the United Nations (FAO). Database on Pesticides Consumption [accessed 2013 Nov 15]. Available from: http://www.fao.org/waicent/FAOINFO/economic/pesticid.htm/.

4. Clark, J.M. and Symington S.B. (2007) Pyrethroid action on calcium channels. Neurotoxicological implications. Invert. Neurosci., 7, 3-16.

5. Nagata, K., Song, J.H., Shono, T. and Narahashi T. (1998) Modulation of the neuronal nicotinic acetylcholine receptorchannel by the nitromethylene heterocycle imidacloprid. $J$. Pharmacol. Exp. Ther., 285, 731-738.

6. Abdallah, F., Fetoui, H., Fakhfakh, F. and Keskes, L. (2012) Caffeic acid and quercetin protect erythrocytes against the oxidative stress and the genotoxic effects of lambda-cyhalothrin in vitro. Hum. Exp. Toxicol., 31, 92-100.
7. El-Demerdash, F.M. (2007) Lambda-cyhalothrin-induced changes in oxidative stress biomarkers in rabbit erythrocytes and alleviation effect of some antioxidants. Toxicol. In Vitro, 21, 392-397.

8. Fetoui, H., Garoui, E.M., Makni-ayadi, F. and Zeghal, N. (2008) Oxidative stress induced by lambda-cyhalothrin in rat erythrocytes and brain: attenuation by vitamin C. Environ. Toxicol. Pharmacol., 26, 225-231.

9. Fetoui, H., Garoui, E.M. and Zeghal, E. (2009) Lambdacyhalothrin-induced biochemical and histopathological changes in the liver of rats: ameliorative effect of ascorbic acid. Exp. Toxicol. Pathol., 61, 189-196.

10. Fetoui, H., Makni, M., Garouiel, M. and Zeghal, N. (2010) Toxic effects of lambda-cyhalothrin, a synthetic pyrethroid pesticide, on the rat kidney: involvement of oxidative stress and protective role of ascorbic acid. Exp. Toxicol. Pathol., 62, 593-599.

11. Mohany, M., Badr, G., Refaat, I. and El-Feki, M. (2011) Immunological and histological effects of exposure to imidacloprid insecticide in male albino rats. Afr. J. Pharm. Pharmacol., 5, 2106-2114.

12. Tuzmen, N., Candan, N., Kays, E. and Demiryas, N. (2008) Biochemical effects of chlorpyrifos and deltamethrin on altered antioxidativedefense mechanisms and lipid peroxidation in rat liver. Cell Biochem. Funct., 26, 119-124.

13. El-Demerdash, F.M. (2011) Oxidative stress and hepatotoxicity induced by synthetic pyrethroids-organophosphate insecticides mixture in rat. J. Environ. Sci. Health C Environ. Carcinog. Ecotoxicol. Rev., 29, 145-158.

14. El-Demerdash, F.M. (2012) Cytotoxic effect of fenitrothion and lambda-cyhalothrin mixture on lipid peroxidation and antioxidant defense system in rat kidney. J. Environ. Sci. Health $B, 47,262-268$.

15. Tukhtaev, K., Tulemetov, S., Zokirova, N. and Tukhtaev, N. (2012) Effect of long term exposure of low doses of lambdacyhalothrin on the level of lipid peroxidation and antioxidant enzymes of the pregnant rats and their offspring. Med. Health Sci. J., 13, 93-98.

16. Marklund, S. and Marklund, G. (1974) Involvement of the superoxide anion radical in the autooxidation of pyrogallol and a convenient assay for superoxide dismutase. Eur. $J$. Biochem., 47, 469-474.

17. Aebi, H. (1984) Catalase in vitro. Enzymology, 105, 121126.

18. Wendel, A. (1980) Enzymatic Bases of Detoxification. Academic press. 1, 333 .

19. Moron, M.S., Depierre, J.W. and Mennervik, B. (1979) Levels of glutathione, glutathione reductase and glutathione Stransferase activities in rat lung and liver. Biochim. Biophys. Acta, 582, 67-78.

20. Mavis, R.D. and Stellwagen, E. (1968) Purification and subunit structure of glutathione reductase from Bakers' yeast. $J$. Biol. Chem., 243, 809-814.

21. Ohkawa, H., Ohishi, N. and Yagi, K. (1979) Assay for lipid peroxides in animal tissues by thiobarbituric acid reaction. Anal. Biochem., 95, 351-358.

22. Lowry, O.H., Rosebrough, N.J., Farr, A.L. and Randal, R.J. (1951) Protein measurement with the folin phenol reagent. $J$. Biol. Chem., 193, 265-275. 
23. Lobin, K.K., Jaunky, V.C. and Ramesh, V. (2017) A review of pesticide use in EU and African countries and associated polices in Proceedings of 120th The IIER International Conference (2017 Sep 15-16). Port Louis, Mauritius.

24. Kimengsi, J.N. and Muluh, N.G. (2013) A comparative assessment of the effect of climate variations on the crops of the Cameroon development corporation (CDC): adaptation options. Env. Nat. Res. Research, 3, 144-156.

25. Brice, K.N., Patricia, A.F., Norbert, N.T. and Mpoame, M. (2017) Environmental and human health assessment in relation to pesticide use by local farmers and the Cameroon development corporation (cdc), Fako division, South-west cameroon. Eur. Sci. J., 13, 454-473.

26. Gesesew, H.A., Woldemichael, K., Massa, D. and Mwanri, L. (2016) farmers knowledge, attitudes, practices and health problems associated with pesticide use in rural irrigation villages, Southwest Ethiopia. PLOS ONE, 11, e0162527.

27. Damalas, C.A. and Koutroubas S.D. (2016) Farmers' exposure to pesticides: toxicity types and ways of prevention. Toxics, $\mathbf{4}, 1$.

28. Balani, T., Agrawal, S. and Thaker, A.M. (2011) Hematological and biochemical changes due to short-term oral administration of imidacloprid. Toxicol. Int., 18, 2-4.

29. El-Gendy, K.S., Aly, N.M., Mahmoud, F.H., Kenawy, A. and El-Sebae, A.K. (2010) The role of vitamin C as antioxidant in protection of oxidative stress induced by imidacloprid. Food Chem. Toxicol., 48, 215-221.

30. Kapoor, U., Srivastava, M.K., Bhardwaj, S. and Srivastava, L.P. (2010) Effect of imidacloprid on antioxidant enzymes and lipid peroxidation in female rats to derive its No Observed Effect Level (NOEL). J. Toxicol. Sci., 35, 577581.

31. Ahmed, M.M. and Zaki, N.I. (2009) Assessment the ameliorative effect of pomegranate and rutin on chlorpyrifos-ethyl- induced oxidative stress in rats. Nature and Science, 7, 4961.

32. Baş, H. and Kalender, Y. (2011) Chlorpyrifos induced cardiotoxicity in rats and the protective role of quercetin and catechin. GUJS, 24, 387-395.

33. Gultekin, F., Delibas, N., Yasar, S. and Kiline, I. (2001) In vivo changes in antioxidant systems and protective role of melatonin and a combination of vitamin $\mathrm{C}$ and vitamin $\mathrm{E}$ on oxidative damage in erythrocytes induced by chlorpyrifosethyl in rats. Arch. Toxicol., 75, 88-96.

34. Oncu, M., Gultekin, F., Karaöz, E., Altuntas, I. and Delibas, N. (2002) Nephrotoxicity in rats induced by chlorpyrifosethyl and ameliorating effects of antioxidants. Hum. Exp. Toxicol., 21, 223-230.

35. Verma, R.S. and Srivastava, N. (2001) Chlorpyrifos induced alterations in levels of thiobarbituric acid reactive substances and glutathione in rat brain. Indian J. Exp. Biol., 39, 174-177.

36. Gopinath, G., Uvarajan, S. and Tamizselvi, A. (2014) Chlorpyrifos-induced oxidative stress and tissue damage in the liver of Swiss albino mice: the protective antioxidative role of root extract of withania somnifera. IJIRST, 1, 100-104.

37. Soujanya, S., Lakshman, M., Anand, K.A. and Gopala, R.A. (2013) Evaluation of the protective role of vitamin C in imidacloprid-induced hepatotoxicity in male albino rats. J. Nat. Sci. Biol. Med., 4, 63-67.

38. Verma, R.S., Mehta, A. and Srivastava, N. (2007) In vivo chlorpyrifos induced oxidative stress: attenuation by antioxidant vitamins. Pestic. Biochem. Physiol., 88, 191-196.

39. Trevisan, M., Browne, R., Ram, M., Muti, P., Freudenheim, J., Carosella, A.M. and Armstrong, D. (2001) Correlates of markers of oxidative status in the general population. Am. J. Epidemiol., 154, 348-356. 